\title{
RADIUS-TO-FREQUENCY MAPPING IN THE RADIO PULSAR EMISSION MECHANISM
}

\author{
S. E. THORSETT
}

Radio Astronomy, California Institute of Technology

\begin{abstract}
A general feature of many models of the pulsar emission mechanism is that radiation of different frequencies is produced at different altitudes above the polar cap. The broadening of pulse components and increasing separation between components at low frequency is in general agreement with these theories. We review the available average profile and pulsar timing observations and discuss the implications for theories of radius-to-frequency mapping.
\end{abstract}

\section{Introduction}

The prediction that pulsar emission of a given frequency is radiated preferentially at a particular altitude, with high frequency radiation coming from relatively near the surface of the star and low frequencies from farther out, is a common feature of most polar cap models of the pulsar emission mechanism. This "radius-to-frequency mapping," or RFM, provides a simple explanation of the observed frequency dependence of the widths of pulsar components and the separations of components in multi-component profiles. The vacuum-gap model of Ruderman and Sutherland (1975), for example, predicts a power-law dependence of component separations, $\Delta \theta \propto \nu^{-0.33}$, the electron-bremsstrahlung model of Vitarmo and Jauho (1973) predicts $\Delta \theta \propto$ $\nu^{-0.45}$, and the curvature-plasma mode model of Beskin et al. (1988) predicts either $\Delta \theta \propto \nu^{-0.14}$ or $\Delta \theta \propto \nu^{-0.29}$.

Considering the ubiquity of the RFM assumption in the pulsar literature, it is surprising that very little systematic analysis of its observational underpinnings has apparently been carried out. We have begun such a study, reviewing the available data on the frequency dependances of pulsar component separations and widths, and the limits on pulsars emission altitudes which can be set by pulsar timing observations.

\section{Observations}

\section{Frequency dependence of component separations}

The increasing separation of profile components with decreasing observing frequency is the classic piece of observational evidence supporting the general predictions of RFM theories. Many authors (Lyne, Smith, and Graham 1971, Rankin 1983b, Seiber, Reinecke, and Wielebinski 1975, Slee, Bobra, and Alurkar 1987) have investigated this behavior, finding that a simple power-law model

$$
\Delta \theta=A \nu^{\alpha},
$$

where we will call $\alpha$ the separation index of the components, does not adequately fit the data for most pulsars. The traditional solution has been to model the data in terms of two power-laws:

$$
\Delta \theta=\left\{\begin{array}{ll}
A_{\text {low }} \nu^{\alpha_{\text {low }}} & \nu<\nu_{\mathrm{b}} \\
A_{\text {high }} \nu^{\alpha_{\text {bigh }}} & \nu>\nu_{\mathrm{b}}
\end{array},\right.
$$

where the break frequency $\nu_{\mathrm{b}}$ separates the high and low frequency regimes. The pulsars listed by Manchester and Taylor (1977) have low frequency separation indices $-0.08 \leq \alpha_{\text {low }} \leq-0.5$ and break frequencies $135 \mathrm{MHz} \leq \nu_{\mathrm{b}} \leq 1500 \mathrm{MHz}$. Generally the slope is shallower at high frequencies, $\left|\alpha_{\text {high }}\right|<$ $\left|\alpha_{\text {low }}\right|$.

Even this model, however, fails to describe the true complexity of the function $\Delta \theta(\nu)$. Rankin (1983b) was forced to introduce a third power-law. fit at intermediate frequency to model PSR $1133+16$, while acknowledging that this was "almost unquestionably" only an approximation to a smooth curve between high and low frequency power-law asymptotes.

We have carried out an extensive review of the published profiles of a number of pulsars with components traditionally thought to correspond to conal components. The selection criterion was that observations be available over a very wide frequency range, at least from $100 \mathrm{MHz}$ to several $\mathrm{GHz}$. In practice this limits the data set to a handful of the strongest pulsars: PSR 0301+19, PSR 0525+21, PSR 1133+16, and PSR 2020+28 with double profiles, PSR 0329+54 and PSR 2045-16 with triple profiles, and PSR $1237+25$ with a five-component profile. The measurements compiled are the peakto-peak separations between the conal components 
Table 1 Summary of component separation observations.

\begin{tabular}{|crccrcrrr|}
\hline Pulsar & $\begin{array}{c}\text { No. of } \\
\text { Pts. }\end{array}$ & $\begin{array}{c}\text { Freq. Range } \\
(\mathrm{MHz})\end{array}$ & $\alpha$ & $\Delta \theta_{\min }$ & $\chi^{2}$ & $\begin{array}{c}\nu_{\mathrm{b}} \\
(\mathrm{MHz})\end{array}$ & $\alpha_{\text {low }}$ & $\alpha_{\text {high }}$ \\
\hline PSR 0301+19 & 8 & $102.5-2700$ & -0.40 & 2.2 & 0.3 & & -0.30 & \\
PSR 0329+54 & 24 & $102.5-10700$ & -0.95 & 19.8 & 1.0 & 1000 & -0.13 & -0.02 \\
PSR 0525+21 & 16 & $102.5-4900$ & -0.53 & 9.9 & 0.5 & 1390 & -0.21 & +0.06 \\
PSR 1133+16 & 44 & $26.1-10700$ & -0.48 & 4.3 & 0.9 & 970 & -0.26 & 0.00 \\
PSR 1237+25 & 31 & $80-4900$ & -0.52 & 7.9 & 0.8 & 1410 & -0.18 & -0.01 \\
PSR 2020+28 & 19 & $102.5-14800$ & -1.19 & 9.2 & 1.1 & 960 & -0.20 & +0.01 \\
PSR 2045-16 & 27 & $80-4900$ & -0.34 & 7.7 & 1.4 & 1500 & -0.15 & -0.07 \\
\hline
\end{tabular}

Values for $\nu_{b}, \alpha_{\text {low }}$, and $\alpha_{\text {high }}$ are from fits to eq.(2) by Sieber, Reinecke, and Wielebinski (1975). Values of $\psi_{\mathrm{h}}, \alpha_{\text {low }}$, and $\alpha_{\text {high }}$ for PSR $0329+54$ are averages of values for separations $1-3$ and $3-4$.

(the outer-most components in the case of complex profiles). In some cases, authors have not reported separation measurements or have failed to provide an uncertainty in their measurement. In these cases component separations are measured directly from the published profiles, with uncertainties estimated to be one-sigma errors in this measurement procedure. The observations are summarized in the references; a more detailed accounting appears in Thorsett (1991).

We have found that for all seven of these pulsars the component separation eventually tends to a constant value at high frequency. That is, the high frequency asymptotic power-law in :4.(2) has index $\alpha_{\text {high }}=0$. This has led us to propose a phenomenological model of pulsar component separations:

$$
\Delta \theta=A \nu^{\alpha}+\Delta \theta_{\min } .
$$

For each pulsar we have found the values of $A, \alpha$, and $\Delta \theta_{\min }$ which minimize $\chi^{2}$, using standara nonlinear least-squares fitting techniques. The results are summarized in table 1 .

As shown in figure 1, no evidence of a discontinuity is seen in the separation-versus-frequency function. In all seven cases, the continuous model [eq.(3)] fits the data very well, over frequency ranges of up to $400: 1$. While this model is strictly phenomenological, with no physical mechanism proposed, it does avoid the ad hoc requirement of a third, interpolating power law to produce a smooth variation from the low to high asymptotes, and eliminates the equally unmotivated "break frequency" separating the pairs of regions with hypothetically different physics.

\section{Frequency dependence of component widths}

It is generally much more difficult to accurately measure the width of the various pulse components than to measure separations between the peaks of two components. The smaller longitude ranges in- volved mean that similar absolute measurement uncertainties lead to larger fractional errors. Broadening of profiles due both to dispersion and to scattering leads to errors which are first order in width measurements but only second order in separation measurements. These problems severely limit the number of pulsars for which reliable widths are available at low frequency. The partial merging of two or more components often makes the very definition of "component width" problematic at best.

Nonetheless, a number of authors have carried out careful studies of the evolution of component width with observing frequency for a large handful of pulsars (Seiber, Reinecke, and Wielebinski 1975, Rankin 1983b, Kuz'min et al. 1986, Slee, Bobra, and Alurkar 1987). They find that the general behavior of $W(f)$ is quite complex. Some pulsars exhibit "absorption features," which narrow the component width over the wavelength range near one meter for many pulsars (Bartel 1981, Rankin $1983 \mathrm{~b}$ ). Some pulsai's show very steady component widths, and a few have components which broaden with increasing frequency, but the general trend is toward narrower components at high frequency. Most pulsar profile and profile component widths are accurately described by simple power-law models. Rankin (1983b), in particular, has emphasized the unexpected nature of this result, given the inadequacy of power-law models for the description of component separations. If component width and separation variations come from the same physical mechanism (i.e., the spreading of magnetic field lines), it is difficult to reconcile this qualitatively different behavior.

\section{Timing observations}

Accurate measurements of pulse arrival times at multiple frequencies can also provide interesting limits on RFM theories. Aberration and retardation will lead to differential delays between frequencies emitted at different radii; Cordes (1978) used the absence of any measurable deviations from cold 

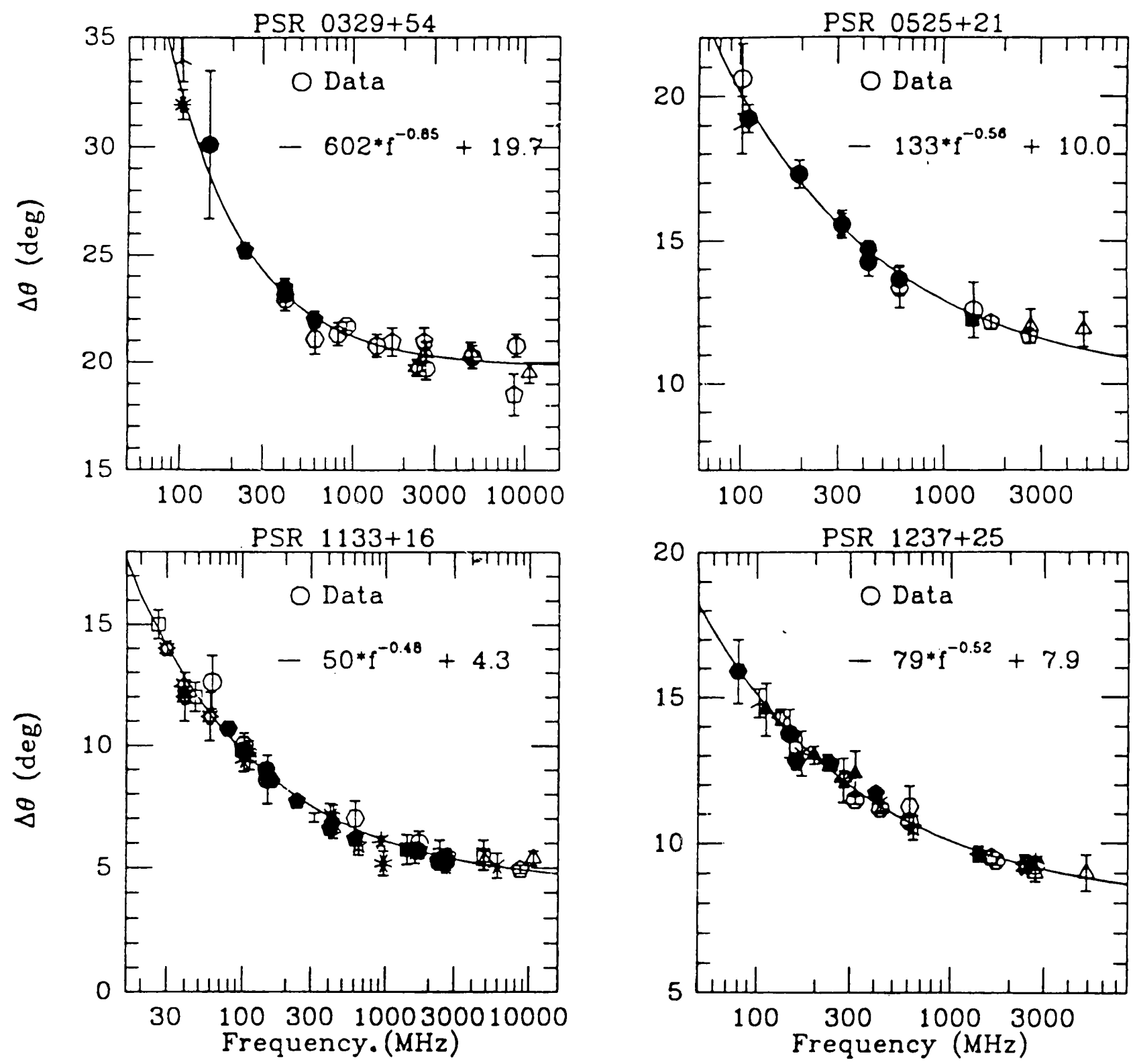

Figure 1 Component separation versus frequency for four pulsars. Solid line is best fit to $\Delta \theta=A \nu^{\alpha}+\Delta \theta_{0}$.

plasma dispersion delays to set limits on emission altitudes in RFM models to less than a few percent of the light-cylinder radius.

Phillips and Wolszczan (1990) have recently extended this technique by including the effects of magnetic field sweepback at high altitudes. They find that for a collection of four pulsars the difference in the altitudes of emission for radiation between 47 and $4800 \mathrm{MHz}$ is less than about $200 \mathrm{~km}$. With the additional assumptions that the field geometry is dipolar and that the pulse width is a measure of the the width of the radiation zone, they constrain the lower edge of the emission range $r_{1}$ (at $4800 \mathrm{MHz}$ ) to be within $r_{1} \sim 10-30 \mathrm{~km}$ from the surface of the neutron star. (Note that this argument assumes RFM: if all frequencies are emitted from a narrow altitude band then timing observations provide no information on the absolute alti- tude of that band.)

Observations of the millisecond pulsar PSR $1937+21$ provide an especially stringent test of emission altitude theories. Cordes and Stinebring (1984) applied the aberration analysis of Cordes (1978) to limit the range of emission from 0.32 to $1.4 \mathrm{GHz}$ to $\Delta r \leq 2 \mathrm{~km}$. Attempts to set a limit on the lower altitude of the lower edge of the emission zone fail, however. Applying the formula of Cordes (1978) and Phillips and Wolszczan (1990)

$$
r_{1} \leq \frac{\Delta r}{\left(\theta_{2} / \theta_{1}\right)^{2}-1}
$$

and using the measured values $\theta_{1}=35 \mu \mathrm{s}$ at $1.667 \mathrm{GHz}$ (Thorsett and Stinebring 1990) and $\theta_{2}=$ $45 \mu s$ at $0.32 \mathrm{GHz}$ (Cordes et al. 1990), we find that $r_{1} \leq 3.1 \mathrm{~km}$, well inside the neutron star! At least one of the three assumptions must be wrong in this 
case: the field may not be dipolar, the relationship between the pulse width and the radiation zone size may depend non-trivially on frequency, or RFM itself may break down. It is possible that millisecond pulsars might differ in some important way from slower pulsars, but recent polarimetry results (Thorsett and Stinebring 1990) indicate that millisecond pulsar emission is qualitatively very similar to that from slower pulsars. At the very least, this result suggests that for PSR $1937+21$ emission occurs very near the surface of the pulsar and implies that measurements of $r_{1}$ for slower pulsars should be treated with caution.

\section{Discussion}

The motivation for RFM models has historically come from theory as well as observation. While details of the pulsar emission mechanism have proven very difficult to come by over the past two decades, RFM seems to arise naturally from the relatively well understood structure of the dipole magnetic field. RFM removes the problem of explaining why different frequencies are emitted preferentially at different distances from the magnetic pole. It must be stressed, of course, that the RFM postulate in a sense only pushes the problem into the third dimension: it is now necessary to explain the emission altitude versus frequency relationship.

Independent of the particular mapping, however, a few general predictions arise. Models of RFM discussed in the literature are generally restricted to highly central line-of-sight trajectories. It is worthwhile to consider the more general case of non-central lines-of-sight. Assume that the diameter of the emission cone varies as some (arbitrary) function of frequency, $\Delta \theta(\nu)$. Then if the intercept angle between the magnetic pole and the line-ofsight is $\beta$, the observed width of the emission cone will be

$$
\Delta \theta^{\prime}=\Delta \theta \sqrt{1-(2 \beta / \Delta \theta)^{2}} .
$$

Thus a non-vanishing $\beta$ leads generally to a steepening of the separation index with higher frequency, not the flattening which is observed. In the extreme case, we expect that two separated components will merge at higher frequency and then leave the beam entirely.

Non-central lines of sight will play an important role whenever $\beta>\Delta \theta / 2$. This is not a rare occurrence: for example Lyne and Manchester (1988) find, from polarization measurements, that for PSR $1133+16 \beta=3.7^{\circ}$, compared with $\Delta \theta \sim 5^{\circ}$ at high frequency. The absence of this predicted behavior is thus a serious challenge to RFM theories.

One of the central tenants of the RFM assumption is that the observed profile widths are closely related to the size of the radiation zone. Cordes (1978) gives the pulse width for a dipole field as

$$
\Delta \theta_{p}=3\left(r / r_{\mathrm{LC}}\right)^{1 / 2},
$$

where the light cylinder radius is $r_{\mathrm{LC}}=c P / 2 \pi$. For PSR 1133+16, given the limit on the high frequency emission radius $r<40 \mathrm{~km}$ (Phillips and Wolszczan 1990 ), we find $\Delta \theta_{p}<4.6^{\circ}$, slightly smaller than observed. If we assume a similar emission radius for PSR $0329+54$ we find $\Delta \theta_{p}<5.9^{\circ}$, much too small to account for the $20^{\circ}$ widths observed at $10 \mathrm{GHz}$.

\section{Summary and Conclusions}

Since its introduction, RFM has found a natural home in many different theories of the pulsar emission mechanism, and has become a part of the "standard model." It is well motivated in the sense that it arises fairly naturally from theoretical arguments which are not highly dependent on the details of the pulsar emission mechanism, and successfully accounts in a general way for component and profile broadening. Unfortunately, like many other aspects of pulsar emission theory, RFM does not account simply for the large variations in behavior seen from pulsar to pulsar. Arguments for the separation of profile components which are based on very general assumptions should lead naturally to very general conclusions.

In detail, RFM models raise as many questions as they attempt to resolve. The trend to a constant component separation at high frequency must be accounted for, as must the variety of separation indices observed at low frequency. The qualitatively different behavior of component separations and component widths is difficult to understand, as is the absence of steepening separation indexes for non-central lines of sight. The very central assumption that the width of the radiation pattern is tied to the width of the polar cap is troubled by the the small emission altitudes required to accommodate timing observations, which predict polar caps much smaller than many observed profiles.

The widespread assumption of RFM arises, of course, from its very usefulness, and until a rival model appears it is the best physical picture that we have. It is important, however, that theoretical work not lose sight of its limitations, as well as successes, in explaining the observational data.

Acknowledgment: This work was supported in part by a National Science Foundation Graduate Fellowship. The author wishes to thank Dan Stinebring for numerous helpful conversations, and Tony Phillips for sharing his collection of pulse profiles prior to publication. 